\title{
Cultura organizacional e innovación en la orientación al mercado de empresas familiares de Pasto (Colombia)
}

\author{
Organizational culture and innovation in the market orientation of family \\ companies of Pasto (Colombia)
}

\author{
Claudia Magali Solarte Solarte ${ }^{1 *}$, Martha Lida Solarte Solarte ${ }^{2}$, \\ José Fernando Barahona Vinasco ${ }^{3}$ \\ ${ }^{1}$ Universidad Cesmag, Colombia \\ ${ }^{2}$ Corporación Universitaria Minuto de Dios Regional Pasto, Colombia \\ ${ }^{3}$ Universidad de Manizales, Colombia
}

Recibido el 20 de octubre de 2017; aceptado el 20 de noviembre de 2018

Disponible en Internet el: 23 de noviemnre de 2018

\section{Resumen}

El propósito de la investigación fue medir la cultura organizacional con la determinación de la capacidad de innovación y reconocimiento del nivel de orientación al mercado que presentan las empresas familiares de la ciudad de San Juan de Pasto (Departamento de Nariño, Colombia). Se trabajó en el año 2016 con una muestra de 234 personas entre gerentes, fundadores y dueños de empresas familiares, tratando con una población infinita. Como metodología se acogió un enfoque cuantitativo, método empírico-analítico y el tipo de investigación explicativo. Así, los resultados evidenciaron que la innovación determina en mayor medida a la orientación al mercado que la cultura organizacional de la empresa; la orientación al mercado es la variable que más causalidad presenta sobre la variable innovación. Una conclusión relevante fue que a medida que la empresa incluye en su producción algún tipo de innovación, la orientación al mercado crece en una magnitud en específico, es decir, las dos variables están estrechamente relacionadas y mantienen una correlación positiva.

Código JEL: M14, M31, O31

Palabras clave: Cultura organizacional; Empresas familiares; Innovación; Orientación al mercado

\footnotetext{
*Autor para correspondencia Correo electrónico cmsolarte@unicesmag.edu.co (C.M. Solarte). La revisión por pares es responsabilidad de la Universidad Nacional Autónoma de México. 


\begin{abstract}
The purpose of the research was to measure the organizational culture with the determination of the capacity for innovation and recognition of the level of market orientation presented by family businesses in the city of San Juan de Pasto (Department of Nariño, Colombia). This study was carried out in 2016 with a sample of 234 people among managers, founders and owners of family businesses, dealing with an infinite population. As a methodology, a quantitative approach, empirical-analytical method and the type of explanatory research were used. Thus, the results showed that innovation determines market orientation to a greater extent than the company's organizational culture; market orientation is the variable that most causality presents on the innovation variable. A relevant conclusion was that as the company includes some type of innovation in its production, market orientation grows by a specific magnitude, that is, the two variables are closely related and maintain a positive correlation.
\end{abstract}

JEL code: M14, M31, O31

Keywords: Organizational culture; Family companies; Innovation; Market orientation

\title{
Introducción
}

Las empresas familiares en Colombia representan un factor fundamental de progreso y empresarismo, Jaramillo (2003) considera que en la actualidad el crecimiento económico del país representado en el PIB se encuentra fuertemente jalonado por la pequeña y mediana industria que generalmente cuenta con accionistas que tienen algún vínculo familiar. Es necesario identificar que las empresas además de generar gran cantidad de recursos por sus tipos de producciones son también una fuente de empleo, tal es la situación, que en la actualidad según el Ministerio de Industria Comercio y Turismo (2018) estas industrias generan el 80\% del empleo en el país, por lo tanto, se vuelve imperiosa la necesidad de concretar la forma mediante la cual estas empresas han logrado establecerse en el mercado y permanecer en él a partir de su orientación al mercado, enfocándose precisamente en su capacidad de innovación y su cultura organizacional. Dadas estas consideraciones el presente documento de investigación pretende resolver la siguiente pregunta: ¿Cómo influye la cultura organizacional y la innovación en la orientación al mercado de las empresas familiares de la Ciudad de Pasto?

Para iniciar es necesario definir que las pequeñas y medianas empresas no solo tienen relevancia al interior de Colombia, esta relevancia se presenta en la totalidad de los países latinoamericanos, según la América Latina Bussines Review (2014) no solo es importante la pequeña y mediana empresa por su generación de trabajo, sino también por los lazos generacionales que crea para darle continuación a las empresas instauradas en el mercado.

De acuerdo con Borroso y Jiménez (2011), se encuentra que la empresa familiar es una organización social en donde convive la empresa y la familia en un sincretismo que genera dos subsistemas superpuestos, interdependientes y generadores de conflictos y soluciones 
empresariales, de la misma manera Soto (2013) afirma que esta es una compañía en donde la toma de decisiones se realiza teniendo en cuenta a una familia que es la que controla la totalidad de procesos productivos, cabe acotar que en esta se tiene muy en cuenta al fundador, el cual como condición sine qua non buscara que sus hijos y nietos sean quienes controlen la empresa en tiempos venideros, con esta intención se promueve también la conservación de las raíces y la unión familiar.

De acuerdo a (Quejada Pérez, Raúl Francisco y Ávila Gutiérrez, Jorge Nelson, 2016,p.4) la temática de las empresas familiares aparece en el contexto actual de los tópicos académicos debido a la importancia que este tipo de compañías tienen dentro de las estructuras de producción de las economías, tanto desarrolladas como en vías de desarrollo. Su importancia radica en el poder generador de empleos que representa, y en el espíritu emprendedor que personifican sus fundadores. No obstante, también se reconocen cuellos de botella dentro de su desempeño, debido al difícil sostenimiento en el tiempo, a causa de las denominadas crisis generacionales. En este sentido, las empresas familiares deben estructurar y establecer estrategias de mitigación de este flagelo, a través de la internacionalización y profesionalización de sus recursos humanos, administrativos y financieros.

Consecuentemente con esta afirmación es necesario argumentar que las empresas familiares en el entorno competitivo son esenciales para la confluencia de recursos y para el mantenimiento de la economía de cualquier país, empero requieren de nuevas investigaciones que les generen conocimientos suficientes para el mantenimiento de estas mismas en el tiempo. Cabe acotar que el mayor problema al que se enfrentan son los cambios generacionales en donde muchas de estas sucumben, para ello se requiere denotar el impacto de la cultura organizacional al interior de las empresas familiares.

En cuanto a la cultura organizacional, es necesario denotar a Hipas (2013) quien considera que esta es el modo de vida propio que las organizaciones han realizado de manera particular, estas refieren a los imaginarios propios de los miembros de la organización es asique se tiene en cuenta los valores, las creencias y los hábitos generales que poco a poco se han instaurado, y que hacen parte esencial del desarrollo de la actividad productiva o comercializadora, un autor que define concretamente la importancia de la cultura organizacional al interior de las empresas familiares es Morales (2010), quien afirma que la cultura como tal es la forma mediante la cual los jefes empresariales denotan su estilo de liderazgo, el autor como tal considera que no es posible evaluar la cultura organizacional sin tener determinado el tipo de liderazgo, esto sobre todo en las empresas familiares, reconociendo en el mismo sentido que esta también es la forma mediante la cual las empresas se relacionan no solo con clientes internos de la organización sino también con los miembros externos a la misma, es así como una empresa puede consolidarse en el mercado como una de las empresas más queridas o más establecidas. A este punto es necesario denotar que Colombia tiene una particularidad 
con respecto a la cultura organizacional, y es precisamente que las empresas son queridas en su entorno, son aceptadas y se consideran como propias.

En la comprensión de la cultura organizacional que impacta a las empresas familiares es necesario tener en cuenta a Monroy (2013), quien afirma de manera vehemente que las empresas como tal son sociedades pequeñas que cuentan con las mismas características organizacionales, es decir, al interior de estas mismas se encuentran normas, conflictos, y jerarquía, es así que estas logran conjugar estos factores y formular una forma de cultura, esta acción se ve todavía más acentuada en las empresas familiares que basan su funcionamiento en las relaciones filiales.

Ahora bien, teniendo en cuenta el otro concepto clave de la presente investigación es el de la innovación otro de los factores que se considera esencial para la supervivencia de las empresas familiares al interior del mercado, inicialmente es necesario tener en cuenta que el concepto de innovación, según Lozano (2010) en la actualidad esta es la capacidad que tienen las empresas para enfrentarse al mundo competitivo a partir de la creación de nuevos productos y servicios, esta le propone a la empresa sorprender al cliente, acudiendo a procesos que están más allá de sus necesidades, pero para ello es necesario acudir a una de las habilidades humanas más vitales: la creatividad.

Según (Avendaño C., W. R., 2012, p.190) las empresas al innovar no solo introducen al mercado un producto, servicio, concepto, idea y/o proyecto, sino que ofrecen una alternativa llamativa e interesante para la satisfacción de las necesidades de las personas (valor), quienes encuentran en el mercado una gran variedad de ofertas y se convierten en agentes que exigen cambios, piden transformaciones y esperan innovación.

Como se puede denotar la importancia en el mercado está relacionada directamente con la forma como confluyen la totalidad de los factores productivos incluyendo en este la cultura organizacional en la producción, para satisfacer, e inclusive ir más allá, a las necesidades del cliente, que a la larga es el elemento esencial en la cadena de valor, en consecuencia, la innovación debe conjugarse con el liderazgo, concentrarse en el cliente y transformar las formas de producción para generar cambios en la sociedad, de esta manera se transforma la empresa a través del conocimiento adquirido y éste se vierte nuevamente en el mercado, transformándolo, a su vez, en una nueva realidad que modifica la actuación de la empresa.

Según Foss y Saebi (2017) la innovación tiene una particularidad que genera competitividad al interior de la organización, y es la creación de valor sostenible, esta como proceso ayuda a que la empresa sea reconocida en un futuro y se mantenga en un mercado competitivo y voraz. Continuando con este análisis es necesario traer a colación a Caoa, Lanlan, Navareb, Jyoti y Jin, Zhongqi (2018) quienes siguiendo la ruta de las nuevas innovaciones para el mercado local, logran concretar que la contratación y promoción del talento local se consideró fundamental para las empresas, esto evidentemente hace parte del conocimiento 
que las empresas familiares tienen al interior de su actuación, ellos sustentan que como el personal local entiende mejor el mercado local que los empleados expatriados, es necesario atraer el talento local y crear condiciones de trabajo favorables para ellos, dado a que esto ayuda muchas de las empresas a obtener una visión superior del mercado local. Las empresas pudieron utilizar un conocimiento más cualitativo de las preferencias del consumidor local y capturar el valor a través de innovaciones de productos / servicios o procesos nuevos.

Ahora bien, a partir de la conceptualización realizada con anterioridad es conveniente afirmar que el hecho de que el cliente interno reconozca el mercado para el cual va a producir genera per se una orientación al mercado, en si Jiménez (2010) denota que la orientación al mercado es un tipo de inteligencia organizacional que considera las necesidades presentes y futura del mercado, coordina las acciones de la totalidad de empresa y hace que confluyan los factores de producción en un mismo fin.

Ynzunza e Izar (2013) reflexionan acerca de las aptitudes que se derivan de la orientación al mercado y plantean que es una fuente de ventaja competitiva con un efecto sobre el desempeño financiero y del mercado, de esta manera prueban que efectivamente las empresas que están orientadas al mercado tienen un desempeño significativamente mayor que aquellas empresas con menor orientación, especialmente en factores que se relacionan a la rentabilidad obtenida por la empresa, la adaptabilidad de la misma ante los cambios en el entorno, la satisfacción de las necesidades y deseos del cliente, etc.

Lo anterior permite subrayar que la orientación al mercado, enfocada principalmente al comportamiento, hace visible que la inteligencia generada puede desprenderse de diferentes actitudes y maneras de comportamiento de los empleados y solventar, de manera oportuna y anticipada, las necesidades que existen día a día en el mercado.

La inteligencia de mercado tiene mucho que ver con la orientación al mercado, es decir, resulta de total importancia que las empresas y organizaciones coordinen todas sus áreas funcionales con el objetivo primordial de desarrollar una cultura que permita crear comportamientos entre los empleados, dirigidos específicamente a las necesidades de los clientes, o sea, que se interesen cada vez más por buscar la forma de brindar mayor satisfacción al cliente y a sus necesidades.

Según Rincón, L. D.; Rojas Berrio, S. \& Montoya, A. (2013) que realiza un análisis de gran cantidad de autores se plantea que "La orientación al mercado se encuentra concebida dentro de una filosofía de mercado que impacta de manera positiva a los integrantes de una organización, con el único fin de cumplir los objetivos y alcanzar los resultados propuestos" (p. 67), de esta manera se comprende que la orientación de mercado debe concebirse como una forma de producción, y de comercialización, las empresas como tal deben percibir la relevancia de estas mismas y concretar al interior de sus acciones y su cultura organización la intención de cumplir con las necesidades del cliente. 
De acuerdo a (Castellanos Ordoñez, Germán y Solano Arboleda, Daniel, 2017, p.92) la relación positiva existente entre la orientación al mercado y los resultados de las empresas. Las empresas que de una u otra forma logran conocer y actuar sobre su mercado tienen un desempeño superior, creando una ventaja competitiva sobre sus rivales. La orientación al mercado es una capacidad desarrollada por las empresas para enfrentar de manera exitosa la competencia, los gustos cambiantes y cada vez más exigentes de sus compradores, la globalización y los rápidos cambios en la economía local y mundial. La cultura organizacional y los procesos operativos requeridos en el desarrollo de la orientación al mercado como una capacidad estratégica dan a las empresas que la tienen una ventaja competitiva frente a sus competidores. En el siglo XXI, las condiciones del mercado son diferentes a las de hace unos 30 años cuando se introdujo el concepto de orientación al mercado; en esa época la gran mayoría de las empresas eran manufactureras y estaban ubicadas en países desarrollados. En el mercado se tienen varias tendencias que vale la pena mencionar y estudiar en un futuro; estas son: 1) las empresas de servicios son cada día más importantes en la economía de los países; 2) la manufactura mundial se ha desplazado a países en vías de desarrollo por sus bajos costos; China e India se han convertido en jugadores muy importantes en el ámbito mundial, y 3) las tendencias mundiales en aspectos del medio ambiente y responsabilidad social empresarial han dado curso a múltiples organizaciones sin ánimo de lucro. En la revisión bibliográfica realizada cabe anotar que las empresas de servicio y organizaciones sin ánimo de lucro no cuentan con un volumen importante de estudios empíricos a nivel mundial. En este sentido, es a partir de 2010 que se observa un crecimiento en los estudios empíricos realizados en países como China e India sobre la relación entre la orientación al mercado y los resultados de las empresas. Estas nuevas tendencias mundiales abrirán nuevos caminos de investigación en la implementación de la orientación al mercado y los resultados de las empresas que lo implementen.

\section{Metodología}

Para la realización del presente documento de investigación de acogió un enfoque cuantitativo, método empírico-analítico y el tipo de investigación explicativo. Como instrumento de recolección de información se estructuró una encuesta que fue aplicada en el año 2016, para ello de manera a priori se hizo un muestreo por selección intencionada o muestreo de conveniencia que, según Casal y Mateu (2003): “Consiste en la elección por métodos no aleatorios de una muestra cuyas características sean similares a las de la población objetivo. En este tipo de muestreos la representatividad la determina el investigador de modo subjetivo" (p. 5), concibiendo finalmente un muestreo con 234 personas, entre gerentes, fundadores y dueños de empresa familiar, se especifica que la población es infinita. 
Con base en el trabajo de Jiménez (2010), se procedió a la utilización del instrumento de medición planteado para determinar el grado de orientación al mercado de las entidades, a partir de los siguientes tres ítems: 1) orientación al cliente, en donde se recogen aquellas características particulares de las empresas que muestran un interés por satisfacer las necesidades y deseos de los clientes; 2) orientación a la competencia, la cual consiste en revisar los mecanismos que las empresas utilizan para estudiar y analizar otras organizaciones similares; 3 ) coordinación inter-funcional que se refiere al análisis de la información que se recolecta y que es útil para cada una de las dependencias de la empresa. Se escoge estos factores dado a que se logra denotar al interior de la comunidad nariñense la configuración de estos mismos como parte fundamental de la organización y la acción empresaria.

Para medir la orientación al mercado, se adoptaron dos instrumentos planteados por Jiménez (2010) que trabaja la escala de MKTOR desarrollada por Narver y Slater (1990), y la escala de MARKOR creada por Kholi y Jaworski (1993); éstos actuaron de forma combinada. Así mismo, para medir la cultura organizacional se hizo uso de un modelo de valores en competencia desarrollado por Quinn y Rohrbaugh (1981, 1983), en donde se proponen cuatro tipologías organizacionales: de desarrollo o adhocrática; de meta racional, propia de las organizaciones orientadas al mercado; centrada en procesos internos, denominada jerárquica; y de recursos humanos, característica de empresas que valoran la tradición, el comportamiento, la participación y la confianza, denominada de grupo. En primera medida es necesario denotar que se escoge este instrumento de medición precisamente porque la idea es notar en primera medida si existe un tipo de enfoque que está centrada en la orientación del mercado, aunque reconociendo que muchas de las organizaciones tendrán su foco en la organización interna debido a su condición de empresa familiar, y el sincretismo entre valores filiales y organizacionales, y una opción alterna que es la del factor humano, esta se escoge precisamente porque bajo los preceptos de la familia coexisten gran cantidad de lazos emocionales, estos a su vez definen unos sentimientos alrededor del personal de trabajo.

En cuanto a la cultura organizacional, específicamente de empresa familiar, se midió a través de la utilización de la sub-escala de cultura F-PEC, que evalúa el alcance de la influencia de la familia sobre los resultados de la empresa, elaborada por Astrachan, J. H., Klein, S. B. y Smyrnios, K. X. (2002); esta evalúa el grado en que los valores familiares y los valores empresariales se sobreponen, y evalúa también el compromiso de las empresas familiares.

Para la innovación, se midió a través de la escala desarrollada por Atuahene-Gima (1996) tomada del documento de Osorio (2014) adaptada para los propósitos de la investigación. Todas las medidas de los ítems se hicieron en una escala Likert de 5 puntos, al considerar de igual manera los siguientes indicadores: la novedad del producto para los 
clientes; la novedad del producto para la empresa; ajuste innovación-comercialización; ajuste innovación tecnológica; ventaja del producto; equipos de trabajo interfuncionales; éxito en el mercado y rendimiento del impacto del proyecto.

Con la revisión bibliográfica se logró identificar aspectos teóricos pertinentes sobre cultura organizacional e innovación. Para la determinación de la relación existente entre las variables analizadas, innovación y cultura organizacional, se utilizó el modelo econométrico Mínimos cuadrados ordinarios, que permitió establecer cuál era el comportamiento y la elasticidad que cada una generaba en la variable dependiente, además de su nivel de dependencia, correlación y ajuste.

\section{Resultados}

Inicialmente se realizó una identificación del sector al que pertenecen las empresas familiares de Pasto encuestadas, clasificándolas por sectores económicos, como se muestra en la tabla 1.

Tabla 1

Actividad económica de las empresas familiares de Pasto

\begin{tabular}{lcc}
\hline \multicolumn{1}{c}{ Actividad económica } & Nro. de empresas & $\%$ \\
\hline Alojamiento y servicios de comida & 18 & 8 \\
Comercio y reparación de vehículos & 111 & 47 \\
Educación & 5 & 2 \\
Industria manufacturera & 23 & 10 \\
Información y comunicaciones & 11 & 5 \\
Otras actividades de servicios & 61 & 26 \\
Transporte y almacenamiento & 5 & 2 \\
\hline Total & 234 & 100 \\
\hline
\end{tabular}

Fuente: Elaboración propia

Según la tabla anterior, en la Ciudad de Pasto el sector terciario o comercio es predominante; esto por cuanto en el Departamento de Nariño no se ha desarrollado un sector industrial; según el Anuario estadístico de la Cámara de Comercio de Pasto (2015): "Las actividades económicas en las cuales se establecieron el mayor número de sociedades para el segundo semestre de 2015, fueron: Comercio y reparación de vehículos con 39 sociedades constituidas” (p. 33). 
La modalidad predominante en el Municipio de Pasto es la microempresa (véase tabla 2), según la (Cámara de Comercio de Pasto, 2015, p. 15) para el movimiento por tamaño de empresa y tal como se puede apreciar, el $99 \%$ se clasifican como microempresas y el $1 \%$ como pequeñas empresas; por tanto, el hecho de que exista mayor cantidad de empresas pequeñas en la ciudad y una mínima parte de medianas empresas, explica por qué no puede absorberse toda la oferta laboral existente en la región.

Tabla 2

Tamaño de la empresa familiar de Pasto

\begin{tabular}{lcc}
\hline \multicolumn{1}{c}{ Tamaño } & Frecuencia & $\%$ \\
Gran empresa & 4 & 2 \\
\hline Mediana empresa & 2 & 1 \\
Pequeña empresa & 10 & 4 \\
Microempresa & 218 & 93 \\
\hline Total & 234 & 100 \\
\hline
\end{tabular}

Fuente: Elaboración propia

\section{Factores determinantes de la cultura organizacional}

En las siguientes tablas se hace una revisión de los factores determinantes de la cultura organizacional de las empresas familiares de la ciudad de Pasto, teniendo en cuenta factores relevantes como la influencia de la jerarquía familiar en la toma de decisiones en la empresa o aspectos de la empresa misma como la forma estructural de la organización.

Tabla 3

Factores de cultura organizacional en empresas familiares de Pasto

\begin{tabular}{clcc}
\hline Factor & \multicolumn{1}{c}{ Pregunta } & $\begin{array}{c}\text { Nivel de } \\
\text { significancia }\end{array}$ & Valor \\
\hline \multirow{2}{*}{67} & $\begin{array}{l}\text { Los miembros de la familia están dispuestos a esforzarse más allá de lo que nor- } \\
\text { malmente se espera con el fin de ayudar a que el negocio de la familia tenga éxito. }\end{array}$ & $2,56 \%$ & $0,11 \%$ \\
79 & Lo más importante en la empresa es la estabilidad. & $1,63 \%$ & $1,74 \%$ \\
85 & El factor cohesionador de la empresa es el compromiso con la innovación & $4,30 \%$ & $6,87 \%$ \\
& y el desarrollo. & & \\
86 & El factor cohesionador de la empresa es el logro de las metas y las tareas. & $-0,33 \%$ & $8,62 \%$ \\
87 & El factor cohesionador de la empresa son las reglas y políticas formales. & $-0,76 \%$ & $6,79 \%$ \\
\hline
\end{tabular}

Fuente: Resultados programa estadístico stata 
Al analizar los factores de la tabla 3, es posible denotar que un factor que se considera irrelevante ante la cultura organizacional es aquel que subyace a la pregunta ¿los miembros de la familia están dispuestos a esforzarse más allá de lo que normalmente se espera con el fin de ayudar a que el negocio de la familia tenga éxito?, dado a que cuentan con un nivel de significancia de 2.56 y un valor p de 0.11 , esto lo define como relativamente bajo. Si bien la estabilidad de la empresa (factor 79) es importante, no aporta significativamente al estudio; entonces, empero, variables que giran en torno a esto si son relevantes, demostrado con una elasticidad del 1,63\%. La variable que expresa que el factor cohesionador de la empresa es el compromiso con la innovación y el desarrollo (factor 85), deja de ser significativo en la medida en que se aleja del nivel del 5\% de significancia y se ubica en un 6,87\%; además, la elasticidad que provoca es baja.

Tabla 4

Modelo MCO para cultura organizacional

\begin{tabular}{ccccc}
\hline Factor & Coeficiente & Desviación Típica & Estadístico $t$ & Valor $P$ \\
\hline Constante & $-0,0007665$ & 0,145665 & $-0,005262$ & 0,9958 \\
64 & 0,0476677 & 0,00858016 & 5,556 & $8,33 \mathrm{E}-08$ \\
65 & 0,12044 & 0,0225756 & 5,335 & $2,46 \mathrm{E}-07$ \\
67 & 0,0256218 & 0,0162914 & 1,573 & 0,1173 \\
68 & 0,0406381 & 0,0225756 & 4,954 & $1,50 \mathrm{E}-06$ \\
69 & 0,053668 & 0,0126901 & 4,229 & $3,50 \mathrm{E}-05$ \\
70 & 0,023888 & 0,0114638 & 2,084 & $3,84 \mathrm{E}-03$ \\
71 & 0,0406381 & 0,0131212 & 3,097 & $2,20 \mathrm{E}-03$ \\
72 & 0,0382801 & 0,00790046 & 4,845 & $2,45 \mathrm{E}-06$ \\
73 & 0,0283353 & 0,007322903 & 3,866 & $1,00 \mathrm{E}-04$ \\
74 & 0,0495093 & 0,0093293 & 5,307 & $2,83 \mathrm{E}-07$ \\
75 & 0,0420892 & 0,00870124 & 5,004 & $1,18 \mathrm{E}-06$ \\
76 & 0,0441069 & 0,00870124 & 5,069 & $8,76 \mathrm{E}-07$ \\
77 & 0,0340723 & 0,0104422 & 3,263 & $1,30 \mathrm{E}-03$ \\
78 & 0,0546923 & 0,0102496 & 5,336 & $2,45 \mathrm{E}-07$ \\
79 & 0,0162835 & 0,0119469 & 1,363 & $1,74 \mathrm{E}-01$ \\
80 & 0,0297436 & 0,0100285 & 2,996 & $3,40 \mathrm{E}-03$ \\
81 & 0,0503737 & 0,0120814 & 4,17 & $4,46 \mathrm{E}-05$ \\
82 & 0,064676 & 0,00703581 & 9,192 & $3,85 \mathrm{E}-17$ \\
83 & 0,0455995 & 0,00684986 & 6,657 & $2,40 \mathrm{E}-10$ \\
84 & 0,131914 & 0,0259389 & 5,086 & $8,10 \mathrm{E}-07$
\end{tabular}




\begin{tabular}{ccccc}
\hline Factor & Coeficiente & Desviación Típica & Estadístico $t$ & Valor $P$ \\
\hline 85 & 0,0430477 & 0,0235274 & 1,83 & $6,87 \mathrm{E}-02$ \\
86 & $-0,00331752$ & 0,0189916 & $-0,1747$ & $8,62 \mathrm{E}-01$ \\
87 & $-0,00766613$ & 0,0185022 & $-0,4143$ & $6,79 \mathrm{E}-01$ \\
\hline
\end{tabular}

\begin{tabular}{cccc}
\hline Media de la variable dep. & 3,420584 & D.T. de la variable. dep. & 0,331292 \\
\hline Suma de cuad. Residuos & 3,166108 & D.T. de la regresión & 0,122787 \\
\hline R - Cuadrado. & 0,876192 & R - cuadrado corregido & 0,862632 \\
\hline F $(23,210)$ & 64,61650 & Valor p (de F) & $3,39 \mathrm{e}-82$ \\
\hline Log - verosimilitud & 171,3981 & Criterio de Akaike & $-294,7962$ \\
\hline Criterio de Schwarz & $-211,8685$ & Crit de Hannan - Quinn & $-261,3598$ \\
\hline
\end{tabular}

Fuente: Resultados programa estadístico stata

Las variables que establecen el logro de metas, tareas empresariales, reglas y políticas formales como factores cohesionadores de la empresa (factores 86 y 87) se comportan con una elasticidad negativa, es decir, que mientras estos se fortalecen, la cultura organizacional se ve afectada en un $0,33 \%$ y un $0,76 \%$ respectivamente. Estos dos factores tampoco son significativos en el modelo, como se evidencia en los resultados estadísticos.

En el mismo sentido, un $\beta 0$ negativo demuestra que la variable cultura organizacional está explicada principalmente por los factores mencionados, y que cualquier cambio que se dé por fuera de los mismos, conlleva a un efecto negativo del ítem. Es decir, el modelo incluye todas las variables fundamentales en su determinación, todas aquellas por fuera del modelo; expresadas por el $\beta 0$ harán variar negativamente a la variable dependiente, o sea, a la cultura organizacional.

Esto se puede comparar con las conclusiones obtenidas en la investigación denominada: El proceso de la empresa familiar Polos desde el imaginario social, de (Coral R., A., 2008, p.52), realizada en el Municipio de Túquerres, Departamento de Nariño, en cuanto a clima organizacional concluye que: El ambiente de la fami-empresa se ve trastocado por las dificultades de comunicación y actitudes defensivas frente al conflicto que no permiten acceder al otro para promover la conciliación y el acuerdo (...) además las situaciones estresantes generadas por las dificultades económicas (...) los conflictos familiares permeabilizan el ambiente laboral.

De igual manera no existe para estas empresas un departamento de mercadeo, los miembros de las empresas familiares deben desarrollar: sondeos, chequeo de precios, pautas publicitarias, etc.; que alimentan de conocimiento a los gerentes o propietarios para la toma de decisiones en cuanto aspectos de inversión, compras u otros relacionados con el negocio. En este orden de ideas, la marca del producto, la etiqueta, el empaque, se basan en las exigencias 
del mercado y con los requerimientos de ley como es el caso de los productos alimenticios. Es indiscutible que la visión de los clientes es fundamental y por lo tanto estar enterado de las expectativas de ellos es clave para la toma de decisiones, con esta información se puede sustentar de manera concreta que existe una falta de organización en cuanto a las acciones que realiza cada uno de los miembros de la familia, y por cuanto no existe una estructura que formule las posibilidades de control al interior de la organización, es necesario denotar en la misma medida que esta es una falencia que procura en muchos casos dirimir la posibilidad de mantenimiento de las empresas en el mercado, siguiendo así con los preceptos que expone Corona y Téllez (2011) en donde se explica que los cambios organizacionales bajo las lógicas filiales modifica sobre manera las acciones competitivas.

Así mismo, en la investigación realizada en la Universidad Peruana de ciencias aplicadas a la escuela de empresa, titulada: Estudio de la cultura organizacional de una empresa familiar peruana, y las posibles soluciones para evitar que este sea un obstáculo en el crecimiento estratégico (2016), es conveniente contrastar que la cultura organizacional es uno de los aspectos más importantes de la empresa familiar que desean permanecer en el mercado, empero, otro aspecto que se denota como esencial es el conocimiento del mercado ya que este les permite permanecer en él y ubicarse asimismo en el entorno, ofreciendo a los clientes aquello que ellos necesitan y requieren, en este caso específico se denota una diferencia parcial pues si bien estos factores resultan importantes los más relevantes se encuentran en el logro de metas y tareas empresariales, las reglas y políticas formales también se evidencia que es una variable fundamental, esta a su vez hace parte de la cultura organizacional de manera no tan directa.

De igual forma, en los resultados de la investigación: El clima organizacional como factor de competitividad en las franquicias de comida rápida en Tijuana, c.c. México, realizado por María Virginia Flores Ortiz, Alfonso Vega López y Edgar Armando Chávez Moreno (2015) se determina que: en lo que se refiere a la cultura organizacional adoptada por las empresas de tipo familiar, especialmente del sector del comercio, es posible recalcar la existencia de un buen ambiente y comunicación entre los miembros de la compañía, en el caso de la presente investigación esta puede constituirse al interior del conocimiento e información de reglas y políticas formales, esta es pues la similitud más grande que se encuentra en las dos investigaciones es menester notar que dada esta semejanza es importante determinar a la comunicación y el flujo de información como uno de los factores que más importancia tiene al interior de la empresa de tipo familiar. Además, dentro de este ítem existe la capacidad de adaptarse a los diversos menesteres familiares, a partir de lo cual se hace necesario la formulación de una visión, una misión, unos objetivos, valores y principios que permiten que la empresa siga el rumbo deseado, se proyecte hacia el futuro y establezca sus propósitos y responsabilidades fundamentales a raíz de su funcionamiento, este es un punto que las empresas pastusas no han establecido, pues dada la informalidad de sus organizaciones estas acciones no se han 
implementado en ese sentido la empresa tiene un carácter poco normativo que no les permite tener claros sus objetivos, y estos de manera generalizada son expresados por el jefe de la empresa que a la vez es el jefe del hogar.

También, en las conclusiones de la investigación "Experiencia generacional y transmisión de poder como elementos de la cultura organizacional en empresas de familia de Campeche, México, realizada por Román Alberto Quijano García, Luis Alfredo Arguelles Ma, José Alonzo Sahuí Maldonado y Deneb Elí Magaña Medina (2015) se expresa que: "la cultura empresarial se manifiesta por la forma tan individual en la que el socio fundador ha dirigido la empresa según su propia visión del vínculo empresa-familia, al manifestar que involucrarse en el negocio familiar ha tenido una influencia positiva en su vida", esta es una semejanza que se expresa sobremanera al interior de las empresas pastusas, a partir del instrumento de recolección de información se puede denotar que las empresas familiares, tuvieron en primera instancia un "ancestro" que fue esencial para dar inicio al negocio que se mantiene hasta el momento es decir dada la ventaja competitiva que desarrolla esta persona es que se mantiene la empresa en el tiempo y el mercado. Es necesario acotar que según la investigación que se contrasta "la mayoría de los entrevistados no perciben que su familia y el negocio compartan gran parte de sus valores" y es a este punto en donde se encuentran diferencias sustanciales con las empresas pertenecientes al mercado pastuso, dado a que los miembros del hogar tienen reglas establecidas, y estas son tomadas como fórmulas para plantear los negocios y establecerlos.

Una investigación que sirve de soporte para entender la situación adyacente al municipio de Pasto y la forma de cómo se integran los valores familiares y organizacionales, es la titulada "Ética organizacional aplicada. Estudio de caso de una organización de servicios sociales en España, realizada por Carla Cubillos Vega (2018) en donde se afirma que, se han explorado y expuesto los valores subyacentes en los discursos de las personas que integran una determinada organización, analizando el grado de coherencia de dichos valores con los supuestos valores que declara tener la entidad. Igualmente se ha indagado en la manera en que estos valores se reflejan e inciden en la práctica. Por lo tanto, los valores de la empresa están bastante relacionados con los valores que expresan y practican sus profesionales. Así mismo, se estima que estos valores o guías para la acción son compartidos con bastante consenso por las personas entrevistadas, lo que permitiría sugerir la existencia de una cultura ética bastante compartida, pese a que se encontró a nivel central bastantes falencias de comunicación y relacionales.

Con esta afirmación se puede denotar que existe una extrapolación de los valores familiares y personales hacia la organización como tal, con ello se comprueba que las familias como tal logran generar un sincretismo entre su vida laboral y su vida familiar, ahora bien lo que no se comparte es precisamente los sistemas de comunicación que si se encuentran establecidos como una variable importante al nivel del clima organizacional pues aunque en la investigación de Cubillos (2018) no se presentan en la actual sí. 


\section{Factores de innovación}

En el análisis de la innovación se consideraron los factores 44 a 63 como se puede apreciar en la tabla 5.

Tabla 5

Factores de innovación

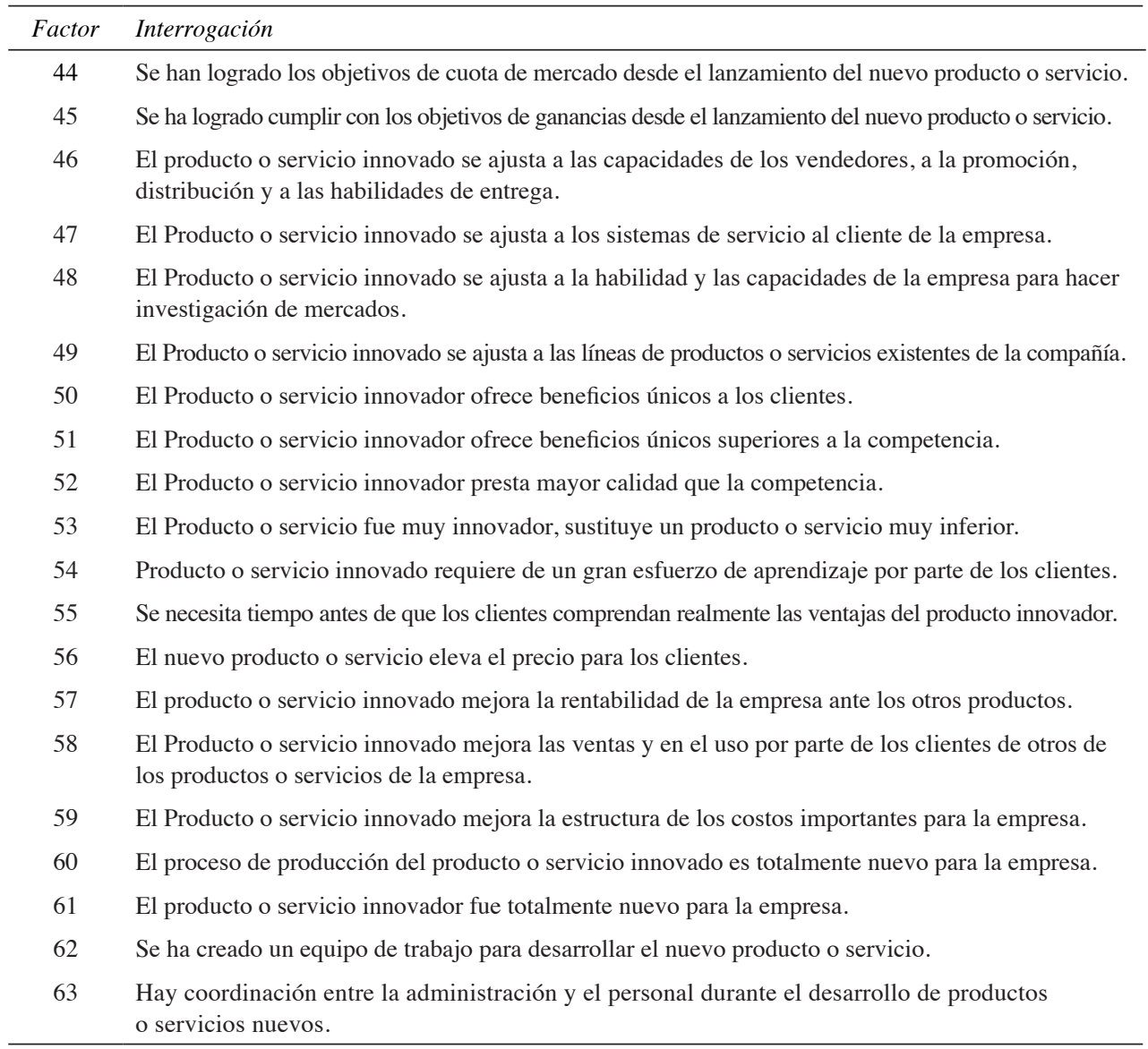

Fuente: Osorio (2014) adaptada para los propósitos de la investigación

A partir de la información de la tabla anterior, se obtuvieron los resultados que se muestran en la tabla 6. 
Tabla 6

Modelo MCO para la variable dependiente innovación

\begin{tabular}{ccccc}
\hline Factor & Coeficiente & Desviación Típica & Estadístico t & Valor $P$ \\
\hline Constante & $-0,0248817$ & 0,0069316 & $-3,59$ & 0,0004 \\
44 & 0,0499182 & 0,00248292 & 19,81 & $3,30 \mathrm{E}-50$ \\
45 & 0,0658098 & 0,00301113 & 21,86 & $2,55 \mathrm{E}-56$ \\
46 & 0,0487128 & 0,00260271 & 18,72 & $7,21 \mathrm{E}-47$ \\
47 & 0,0504111 & 0,00195781 & 25,75 & $2,47 \mathrm{E}-67$ \\
48 & 0,0461041 & 0,00368633 & 12,51 & $2,84 \mathrm{E}-27$ \\
49 & 0,0516423 & 0,00224349 & 23,02 & $1,07 \mathrm{E}+59$ \\
50 & 0,0492073 & 0,00207259 & 23,74 & $9,28 \mathrm{E}-62$ \\
51 & 0,0495248 & 0,00197751 & 26,38 & $4,90 \mathrm{E}-69$ \\
52 & 0,0489909 & 0,00228177 & 21,47 & $3,47 \mathrm{E}-55$ \\
53 & 0,050331 & 0,00210031 & 23,96 & $2,18 \mathrm{E}-62$ \\
54 & 0,046313 & 0,0035868 & 12,91 & $1,49 \mathrm{E}+28$ \\
55 & 0,0526498 & 0,00380805 & 13,83 & $1,87 \mathrm{E}-31$ \\
56 & 0,0439861 & 0,00411943 & 10,68 & $1,36 \mathrm{E}-21$ \\
57 & 0,0547302 & 0,00298086 & 18,36 & $9,05 \mathrm{E}-46$ \\
58 & 0,0517767 & 0,00436102 & 11,87 & $2,77 \mathrm{E}-25$ \\
59 & 0,051226 & 0,00405958 & 12,62 & $1,26 \mathrm{E}-27$ \\
60 & 0,0564071 & 0,00194169 & 29,05 & $4,98 \mathrm{E}-76$ \\
61 & 0,0481216 & 0,00224898 & 21,4 & $5,72 \mathrm{E}-55$ \\
62 & 0,0457773 & 0,00317494 & 12,42 & $2,44 \mathrm{E}-33$ \\
63 & 0,0540115 & 0,00206477 & 26,16 & $1,91 \mathrm{E}-68$ \\
\hline
\end{tabular}

Fuente: Resultados programa estadístico stata

Así entonces, se puede apreciar que todos los factores que integran el ítem innovación, tienen una alta significancia en el modelo ya que están por debajo del 5\% y la variación que provocan sobre la variable innovación es positiva. El factor 45 es el que más aporta a la innovación, referido a la variable que expresa que se ha logrado cumplir con los objetivos de ganancias desde el lanzamiento del nuevo producto o servicio, provocando una elasticidad del $6.58 \%$, seguido de la variable que plantea que el producto innovado es totalmente nuevo para la empresa (factor 60), aporta una elasticidad de 5,64\%. El grado de determinación para este modelo es alto, dado que se ubica en un $99,90 \%$, e indica que la innovación está realmente explicada por cada uno 
de los factores establecidos, esto denota de manera vehemente que la teoría explicada en el presente documento de investigación se logra comprobar en los mercados locales, en este caso específico en el mercado de Pasto.

Los resultados anteriores se contrastan de esta manera con el documento presentado a continuación que perteneces al ámbito internacional, José Luis Esparza Aguilar, Domingo García Perez de Lema y Antonio Duréndez Gómez Guillamón (2009) realizaron la investigación: Gestión estratégica y competitiva de las empresas familiares turísticas mexicanas: Un estudio empírico en la región de Quintana Roo (México). En ella se analizó la forma cómo las empresas familiares hacen uso de diferentes factores de competitividad, tales como la planificación estratégica, la formación y profesionalización de los recursos humanos, así como las herramientas de gestión, contables y financieras, en este caso se denota que los factores esenciales para la innovación tienen su preocupación en el público que hace recepción de los productos o servicios; dado a esto se encontraron algunas debilidades que las empresas familiares deberían subsanar, en este caso específico es iniciar con procesos de innovación constantes en donde se logre coordinar la totalidad de aspectos productivos para generar una mejora en la satisfacción del cliente.

De esa manera, es posible mencionar que las empresas familiares le otorgan gran importancia a la puesta en marcha de una compleja y minuciosa planificación de tipo estratégico, utilizan pocos recursos financieros para la formación del capital humano y no hacen uso frecuente de los instrumentos de gestión y eficiencia, como lo son los registros contables, los diversos análisis económicos, la planificación de orden estratégico y el Balanced Score Card estos comportamientos se ven reflejados en las dos investigaciones, lo que acrecienta los problemas con respecto a la planificación empresarial y no brindan herramientas para realizar innovación en productos y servicios que respondan a las necesidades de la empresa, tampoco es posible realizar un análisis detallado y rápido de la situación financiera de la empresa, pues no se cuenta con un sistema de contabilidad tecnificado, esto se presenta precisamente a la falta de inclusión de diversas tecnologías al interior de la empresa como tal.

Ya una vez determinados por factores esenciales en cada una de las variables se procedió a realizar un modelo de mínimos cuadrados ordinarios, con el objeto de correlacionar las variables del estudio, de la siguiente manera:

\section{Orientación al mercado}

Al ubicar a la variable orientación al mercado como dependiente, y analizar cuál es la influencia de cada una de las variables sobre la misma, se obtuvo lo siguiente: 
Tabla 7

Modelo 2 MCO con orientación al mercado como variable dependiente

\begin{tabular}{|c|c|c|c|c|}
\hline Variables & Coeficiente & $\begin{array}{c}\text { Desviación } \\
\text { Típica } \\
\end{array}$ & Estadístico t & Valor P \\
\hline Constante & 2,81544 & 0,513386 & 5,484 & $1,11 \mathrm{E}-07$ \\
\hline Orientación a la competencia & $-0,00190073$ & 0,0521537 & $-0,03644$ & 0,971 \\
\hline Coordinación interna & $-0,0202647$ & 0,0809295 & 0,2504 & 0,8025 \\
\hline Capacidad de respuesta al mercado & 0,0174199 & 0,086396 & 0,2016 & 0,8404 \\
\hline Generación y diseminación de la información & 0,259273 & 0,0671631 & 2,014 & 0,0452 \\
\hline Resultados del negocio & 0,0304537 & 0,0560802 & 0,543 & 0,5876 \\
\hline Innovación & 0,239985 & 0,0516615 & 4,645 & $5,76 \mathrm{E}-06$ \\
\hline Cultura organizacional & $-0,0407179$ & 0,131823 & $-0,3089$ & 0,7577 \\
\hline Media de la variable dependiente & 3,818376 & \multicolumn{2}{|c|}{ D.T de la variable dependiente } & 0,647168 \\
\hline Suma de cuadrado Residuos & 79,14581 & \multicolumn{2}{|c|}{ D.T. de la regresión } & 0,591779 \\
\hline $\mathrm{R}$ - cuadrado & 0,188968 & \multicolumn{2}{|c|}{ R- cuadrado corregido } & 0,163848 \\
\hline $\mathrm{F}(7,226)$ & 7,522473 & \multicolumn{2}{|c|}{ Valor p (de F) } & $3,18 \mathrm{e}-08$ \\
\hline Log-verosimilitud & $-205,2002$ & \multicolumn{2}{|c|}{ Criterio de Akaike } & 426,4004 \\
\hline Criterio de Schwarz & 454,0429 & \multicolumn{2}{|c|}{ Criterio de Hannan - Quinn } & 437,5458 \\
\hline
\end{tabular}

Fuente: Resultados programa estadístico stata

En la tabla 7 se muestra la importancia de variables como la innovación, la generación y diseminación de la información dentro de la empresa familiar a la hora de decidir sus políticas de orientación al mercado; la primera refleja una elasticidad de la variable dependiente en un $23.99 \%$, teniendo un nivel de significancia demasiado alto. La segunda representa una elasticidad de $25.92 \%$, con un valor p de 4,5\%. Las dos variables conllevan a un cambio positivo en la orientación al mercado.

Por su parte, las variables de orientación a la competencia, coordinación interfuncional y cultura organizacional conllevan a que el factor dependiente tenga una variación negativa. La capacidad de respuesta de la empresa ante cambios en la competencia, representa una elasticidad positiva para la variable dependiente en $1,74 \%$, con un valor p demasiado elevado, lo cual infiere que es una variable poco significativa para la relación.

De manera concreta se puede afirmar que en la orientación al mercado tiene una mayor relevancia y produce cambios positivos significativos, factores como la innovación y la comunicación al interior de la empresa, mientras que de manera concreta las empresas no realizan un seguimiento a las empresas que se consideran competencia, así como tampoco se presenta una cultura organizacional adecuada, estos dos últimos aspectos pueden determinarse como opciones de mejora. 
Tabla 8

Modelo 4 MCO con orientación al mercado como variable dependiente

\begin{tabular}{lcccc}
\hline Variables & Coeficiente & Desviación Típica & Estadístico t & Valor P \\
\hline Constante & 11,28077 & 0,30273 & 1,231 & $3,37 \mathrm{E}-05$ \\
Generación y diseminación de la & & & & \\
información & 0,316954 & 0,0891681 & 3,555 & 0,0005 \\
Innovación & 0,220495 & 0,0531731 & 4,147 & $4,75 \mathrm{E}-05$ \\
\hline
\end{tabular}

\begin{tabular}{llll}
\hline Media de la variable dependiente & 3,818376 & D.T de la variable dependiente & 0,647168 \\
\hline Suma de cuadrado Residuos & 79,33245 & D.T. de la regresión & 0,586029 \\
R - cuadrado & 0,187055 & R- cuadrado corregido & 0,180017 \\
F $(2,231)$ & 26,57610 & Valor p (de F) & $4,09 \mathrm{e}-11$ \\
Log-verosimilitud & $-205,4758$ & Criterio de Akaike & 416,9515 \\
Criterio de Schwarz & 427,3175 & Criterio de Hannan - Quinn & 421,1311 \\
\hline
\end{tabular}

Fuente: Resultados programa estadístico stata

Si se observa la tabla anterior, se identifica que las dos principales variables determinísticas de la variable dependiente, orientación al mercado, son la generación y diseminación de la información entre las distintas dependencias de la empresa familiar, y la innovación que esta adopte para mejorar su competitividad. Las dos variables implican elasticidades positivas de la orientación al mercado y son altamente significativas. El coeficiente de determinación (R cuadrado) para el modelo, es de $18.71 \%$, lo que demuestra que no son las únicas variables que pueden determinar a la variable dependiente, y que inclusive al interior de los modelos de negocios de la ciudad de Pasto confluyen otro tipo de variables que pueden resultar más significativas estas determinadas por un factor independiente $\beta 0$. Dentro del modelo, la variable generación y diseminación de la información explica a la orientación al mercado en un 31,69\%, y la innovación explica la orientación al mercado en un $22,05 \%$ (véase tabla 8 ).

\section{Relación de las variables innovación y cultura organizacional}

Al correlacionar las variables cultura organizacional e innovación para hacer una interpretación de la variable orientación al mercado, se obtuvo el siguiente comportamiento: 
Tabla 9

Modelo 5 MCO con Orientación al mercado como variable dependiente

\begin{tabular}{lcccc}
\hline Variables & Coeficiente & Desviación Típica & Estadístico t & Valor P \\
\hline Constante & 3,32198 & 0,414616 & 8,012 & $5,55 \mathrm{E}-14$ \\
Innovación & 0,287119 & 0,0466608 & 6,153 & $3,32 \mathrm{E}-09$ \\
Cultura organizacional & $-0,0448779$ & 0,131096 & $-0,3423$ & $7,32 \mathrm{E}-01$ \\
\hline
\end{tabular}

\begin{tabular}{lccc}
\hline Media de la variable dependiente & 3,818376 & D.T de la variable dependiente & 0,647168 \\
\hline Suma de cuadrados residuos & 81,71216 & D.T. de la regresión & 0,594754 \\
\hline $\mathrm{R}$ - cuadrado & 0,162670 & R- cuadrado corregido & 0,155420 \\
\hline $\mathrm{F}(2,231)$ & 22,43841 & Valor p (de F) & $1,24 \mathrm{e}-09$ \\
\hline Log-verosimilitud & $-208,9338$ & Criterio de Akaike & 423,8675 \\
\hline Criterio de Schwarz & 434,2335 & Criterio de Hannan - Quinn & 428,0471 \\
\hline
\end{tabular}

Fuente: Resultados programa estadístico stata

Según lo anterior, la innovación es una variable que genera una elasticidad positiva sobre la orientación al mercado, mientras que la variable cultura organizacional genera efectos negativos. La primera vuelve a obtener un alto grado de significancia, criterio con el cual no cumple la segunda variable independiente evaluada. Así, la orientación al mercado se explica por la innovación que se hace dentro de las empresas en tanto la cultura organizacional no tiene los mismos efectos positivos sobre la primera.

Pero si solo se analiza la variable cultura organizacional sin tener en cuenta la innovación en la ciudad de Pasto se tiene la investigación: Análisis de la orientación al mercado y la cultura organizacional de las pequeñas empresas de la industria de artes gráficas local, realizado por Héctor Luis Ávila Baray (2009). En este se afirma que el desempeño organizacional, puede verse permeado por la influencia de la cultura organizacional en la orientación al mercado que posee una empresa y en este caso la situación vivenciada por las empresas familiares pastusas, además se logra concluir que, gracias a los diversos análisis estadísticos (se logra evidenciar) que sí existe relación entre la orientación al mercado y la cultura organizacional que caracteriza a la industria de artes gráficas local. Las empresas que registran mayor orientación al mercado se caracterizan por una cultura organizacional emprendedora y en aquellas con menor orientación al mercado predomina la cultura organizacional de consenso, es decir, de conformidad entre los miembros que integran la empresa. 
Ahora bien, contrastando los resultados de la investigación analizada con la presente investigación es conveniente afirmar que en sí las empresas encuentran en la innovación la motivación para lograr conjugar los miembros de la familia en los entornos organizacionales.

Según Sixto (2010), la innovación es el factor por medio del cual se logra satisfacer las necesidades del mercado, es así como las empresas logran mantenerse en los algoritmos conceptuales de los clientes y ser aceptadas por los consumidores, es asique descubren las necesidades del cliente y las hacen realidad, empero para ello se hace necesario que la totalidad de los agentes vinculados con la empresa generen cada uno en sus dependencias procesos de generación de información adecuada, la idea es aquí desde cada una de las variables del proceso productivo sorprender al cliente.

Respecto a la cultura organizacional y su relación con la innovación, Terzaghi (2010) expresa que es necesario que las empresas generen situaciones y un ambiente de trabajo que beneficie la creación de nuevos productos y adopte nuevas tecnologías. Para esto es de importancia la colaboración y el compromiso del talento humano involucrado en los proyectos de la empresa y más cuando se trata de la familia, que tiene al interior de su funcionamiento establecidas normas y reglas, este es evidentemente una conceptualización que en la actualidad se está generando por las empresas familiares pastusas.

Tabla 10

Examen de causalidad 1

\begin{tabular}{lccc}
\hline Null Hypothesis & Observaciones & F-statistic & Probabilidad \\
\hline INNOVACIÓN does not granger cause ORIENTACIÓN & & & \\
AL MERCADO & 232 & 3,77680 & 0.0243 \\
ORIENTACIÓN AL MERCADO does not granger cause & & 3,94927 & 0.0206 \\
INNOVACION & & & \\
CULTURA ORGANIZACIONAL does not granger cause & & & \\
ORIENTACIÓN AL MERCADO & 232 & 3,16799 & 0.0440 \\
ORIENTACIÓN AL MERCADO does not granger cause & & 0,21048 & 0.8104 \\
CULTURA ORGANIZACIONAL & & & \\
CULTURA ORGANIZACIONAL does not granger Cause & & 0,66835 & 0.5136 \\
$\begin{array}{l}\text { INNOVACION does not granger cause } \\
\text { CULTURA ORGANIZACIONAL }\end{array}$ & 232 & 1,51486 & 0.2221 \\
\hline
\end{tabular}

Fuente: Resultados programa estadístico stata

En la evaluación de la relación de causalidad que tienen las variables, mostrada en la tabla 10, se puede observar que las hipótesis nulas planteadas, son las siguientes: 
- La orientación al mercado no es causada por la innovación y viceversa.

- La cultura organizacional no es causada por la orientación al mercado y viceversa.

- La innovación no es causada por la cultura organizacional y viceversa.

Dados los resultados es conveniente afirmar que en general no existe una relación de causalidad entre las variables del estudio es decir que su actuación se considera independiente y basada en factores disimiles, sin embargo, cuando se excluyen aquellas variables que se consideran poco significativas se tiene los siguientes resultados:

Tabla 11

Examen de causalidad 2

\begin{tabular}{lccc}
\hline Null Hypothesis & Observaciones & F-statistic & Probabilidad \\
\hline $\begin{array}{l}\text { INNOVACIÓN does not granger cause } \\
\text { ORIENTACIÓN AL MERCADO }\end{array}$ & 232 & 3,77680 & 0.0243 \\
$\begin{array}{l}\text { ORIENTACIÓN AL MERCADO does not granger } \\
\text { cause INNOVACION }\end{array}$ & 232 & 3,94927 & 0.0206 \\
\hline
\end{tabular}

Fuente: Resultados programa estadístico stata

En la tabla anterior es evidente que la innovación, además de ser una variable altamente significativa en el modelo, también es causal de la orientación al mercado, dado que se rechaza la hipótesis nula, con un nivel de significancia relevante. La orientación al mercado también es explicativa de la innovación, siendo su probabilidad un poco más alta que la anterior. Estos resultados pueden ser contrastados con los de Michaels, Eric T. y Boecker, Andreas (2017) en su documento titulado: "Competitive strategies among Ontario farms marketing direct to consumers", en donde se destacan los siguientes resultados: "se ha encontrado que una orientación empresarial está asociada positivamente con la intensidad de la innovación tanto del producto como del marketing, lo que sugiere que las empresas proactivas e innovadoras tienen más probabilidades de participar en el desarrollo de nuevos productos o implementar cambios en la comercialización de los productos" esta es pues la situación que al momento están presentando las empresas de la ciudad de Pasto, también una orientación de mercado está asociada positivamente con la innovación de productos y marketing, en este caso se reconoce en primera medida que las empresas que laboran en la actualidad en las empresas por ser de tipo familiar son personas que tienen una amplia relación con el mercado local y con las necesidades de la población como tal esto les permite otorgar los productos precisos que necesita 
la comunidad, ahora bien también es conveniente afirmar que las diferentes áreas de la empresa se encuentran apalancadas muchas veces por las mismas personas esto le da continuidad al proceso y genera mercancías mayormente complementadas.

\section{Conclusiones}

A partir del estudio realizado para la ciudad de Pasto, es necesario acotar en primera medida que la actividad productiva y comercial que en mayor medida están realizando los empresarios es el comercio y la reparación de vehículos, asimismo se denota que la mayoría de las empresas que se encuentran constituidas como tal tienen la calidad de micro, y pequeña empresa esto contento con un porcentaje de $97 \%$, con esta afirmación se puede evidenciar que Colombia maneja las mismas lógicas productivas que el resto de América Latina, en donde la incidencia de la Mi pymes también es significativa.

En el análisis concreto de las variables logra identificarse que las empresas familiares de la ciudad de Pasto a la actualidad no presentan reglas y políticas formales, lo que dificulta mucho la división de tareas y la acción coordinada de actividades, en el mismo sentido tampoco existe una fijación de logros a largo, mediano y corto plazo es asique muy pocas veces se presenta una organización adecuada de tiempos y espacios de acción, dados estos factores se denota la presencia de una baja competitividad, sostenibilidad y expansión de los mercados, se concreta de esta manera que existe correlación directa entre la teórica planteada por Corona et al (2003) y la realidad de la región.

Es necesario hacer evidente a este punto que uno de los factores que dirimen el riesgo de cierre de las MIPYMES en Pasto es la innovación, recordando de esta manera el factor de correlación de las dos variables, dado a que esta genera per se una acción significativa sobre la orientación al mercado, le permite así establecerse en este con productos que llegan a solucionar las problemáticas específicas de la población y a mejorar significativamente los ingresos de las organizaciones, permitiéndoles así mantenerse en el tiempo, cabe acotar que los procesos de innovación son llevados a cabo por los miembros jóvenes de las familias. Es necesario comprender de esta manera que la orientación al mercado es la variable que más causalidad presenta sobre la variable innovación para las empresas analizadas esto con un coeficiente de correlación de $87 \%$.

Teniendo en cuenta a Cortes et al (2010) se logra evidenciar que como tal las organizaciones que propenden al interior de sus procesos de innovación la satisfacción de los clientes, son aquellas que sobreviven al tiempo y más si se trata de empresas familiares, evidentemente este es entonces el punto fuerte que a la actualidad está manejando la empresa pastusa. Ahora bien, esta puede considerarse una forma de establecer mejores condiciones empresariales y dirimir la posibilidad de quiebra que 
tienen en la actualidad las organizaciones, es necesario acotar que en la actualidad subsisten diferentes problemas que están relacionados con la competitividad y esta es una forma de acrecentarla.

De acuerdo al estudio, por los resultados de la baja orientación al mercado e innovación de las empresas familiares, es necesario desarrollar un proceso de sensibilización y formación, como objetivo global en el proceso de formación académica, con experiencias directas en el campo empresarial, que permita la toma de decisiones acertadas y evaluación de las oportunidades del entorno, esto en si se representa como una forma de cualificación del talento humano pero también simboliza el apoyo al entorno familiar.

En lo que respecta a la innovación que precede de la inclusión de nuevas formas de producción y comercialización, en la región se nota un bajo nivel de incorporación tecnológica; este hecho se explica por la escasa inversión tanto pública como privada que históricamente registra el Departamento de Nariño. Esta condición puede ser disminuida significativamente si se presenta una orientación al mercado adecuada.

De acuerdo a (Solarte, C. Solarte, M. Arcos, C., 2017, p. 26) es de total importancia, que así como existen diferentes programas que ayudan a capacitar y a direccionar a las personas y a la creación de nuevas empresas, se hace necesario que existan también diversos programas o políticas de acompañamiento que ayuden a encaminar los procesos con respecto a temas empresariales como de orientación al mercado, innovación, cultura organizacional; estos tres ítems son recursos de carácter estratégico para un mejoramiento significativo de los resultados del negocio.

Desde la perspectiva de los autores y bajo la mirada preocupante de las acciones empresariales en la ciudad se hace relevante denotar que deben formulares planes consientes que generen un nivel real de innovación para romper con paradigmas comportamentales en la población como tal, quienes en su mayoría opinan que en la actualidad las empresas regionales no cumplen con sus expectativas, es necesario aclarar que esta situación se produce precisamente porque los mercados regionales se encuentran abarrotados de productos que no son producidos al interior de la región, y que tienen especificaciones diferentes.

Se requiere en la misma medida determinar de manera fehaciente las variables que denotan una incidencia al interior de la cultura organizacional, pues si bien las empresas pastusas se tienen como amables cumpliendo los preceptos de la Amerizan Bussines Review (2010) se requiere que esas sean competitivas para que se mantengan en el mercado.

Consecuentemente, se entiende que el jefe de familia es generalmente el direccionado de la empresa, y esto crea en muchas ocasiones procesos de inconformismo al interior de la acción productiva, para evitar estos conflictos se requiere entonces iniciar procesos organizativos adecuados que permita tomar a los miembros de la familia un nivel jerárquico importante y un empoderamiento adecuado. 
En general la competitividad, la innovación y la cultura organizacional si bien son variables que aparentemente no tienen relación entre sí, si se consideran variables considerablemente relevantes para que una empresa se mantenga en el paso del tiempo, es por ello que se requiere de una mayor cantidad de atención desde las organizaciones medianas y pequeñas en la ciudad de Pasto, incluyendo así estas como ejes procedimentales esenciales en la determinación de sus actividades productivas.

\section{Referencias}

Alvarado, O. y Monroy, R. (2013). Cultura organizacional en una empresa propiedad de sus trabajadores. Cuadernos de Administración, 26 (47), 259-283. Disponible en: http://www.scielo.org.co/pdf/cadm/v26n47/v26n47a11.pdf

América Latina Business Review (2014). Empresas de familia en América Latina. Disponible en: http://www.businessreviewamericalatina.com/leadership/134/Empresas-familiares-en-Am\%C3\%A9rica-Latina Consultado: $15 / 04 / 2016$.

Azuela Flores, J. I. \& Jiménez Torres, N. H. (2014). El estado del arte de la orientación al mercado en las organizaciones culturales. Contaduría y Administración. 59 (1), 259-282. https://doi.org/10.1016/S0186-1042(14)71251-3

Becerra R., F.; Álvarez G., Claudia. (2011). El talento humano y la innovación empresarial en el contexto de las redes empresariales: el clúster de prendas de vestir en Caldas-Colombia. Estudios gerenciales. 27 (119), 209232 Disponible en: http://www.scielo.org.co/pdf/eg/v27n119/v27n119a11.pdf

Becerra R., F.; Serna, H. \& Naranjo V., J. (2013) Redes empresariales locales, investigación y desarrollo e innovación en la empresa. Clúster de herramientas de Caldas, Universidad ICESI. Colombia. 29 (127), 247-257. https://doi.org/10.1016/j.estger.2013.05.013

Cámara de Comercio de Pasto. (2015). Anuario estadístico: Movimiento del registro público. Pasto: Ediciones de la Cámara de Comercio.

Cauzo Bottala, L. \& Cossío Silva, F. J. (2012). Análisis del efecto inmediato y diferido de la orientación al mercado sobre los resultados organizacionales. Un estudio longitudinal. Investigaciones Europeas de Dirección y Economía de la Empresa. 18 (3), 228-236. https://doi.org/10.1016/j.iedee.2012.05.002

Cobian Media (2014). Cómo crear una cultura organizacional de innovación. Disponible en: https://www.cobianmedia.com/2014/01/29/como-crear-una-cultura-organizacional-de-innovacion/ Consultado: 16/02/2016

Corona, J. y Téllez Roca, J., (2011). El protocolo familiar, en Empresa Familiar: aspectos jurídicos y económicos, ed. por J. Corona, Ediciones Deusto: Barcelona.

De la Torre A., Conde, J. y Antón, C (2015). Concepto y naturaleza de la empresa familiar. Disponible en: http:// www.laboral-social.com/concepto-naturaleza-empresa-familiar.html Consultado: 10/03/2016.

Flores Ortiz, M. V. Vega López, A. Chávez Moreno, E. A. (2015). Factores de Contingencia Que Inciden en la Profesionalización de las Empresas de familia del Sector Comercio en Tijuana, B.C., México. Revista Internacional Administración \& Finanzas RIAF 8 (3), 15-29. Disponible en: https://ssrn.com/abstract=2660363

Fuentes D., A. E. (2012). La cultura organizacional: factor clave de éxito en las empresas del siglo XXI. Tesis pregrado Ingeniería Industrial, Universidad de Cartagena.

Gutiérrez, M. (2013). La cultura organizacional, variable importante para obtener la ventaja competitiva. Tesis posgrado alta gerencia, Universidad Militar Nueva Granada.

Halpern, N. (2010). Marketing innovation: Sources, capabilities and consequences at airports in europe's peripheral areas. Journal of Air Transport Management, 16 (2), 52-58. https://doi.org/10.1016/j.jairtraman.2009.10.002

Jansa, S. (2010). Manual de Oslo - Innovación. Universidad Nacional de Educación a Distancia (UNED). Disponible en: http://portal.uned.es/portal/page?_pageid=93,23280929\&_dad=portal Consultado: 1/03/2016. 
Kotler, Philip \& Armstrong, Gary. (2013). Fundamentos de marketing. México: Pearson.

Kotler, Philip \& Armstrong, Gary. (2016). Fundamentos de marketing, productos, servicios y marcas. Parte 1 : Definición de Marketing y del Proceso de Marketing, pp. 48-49. México: Pearson.

Macías R., Víctor H., D. E. (2017). Sucesión en empresas familiares. Análisis desde la teoría de la agencia. Caso: Caldas. Criterio Libre, 9 (15), 193- 212. https://doi.org/10.18041/1900-0642/criteriolibre.2011v9n15.1208

Medrano S., N. (2012). Factores determinantes de la adopción de innovaciones en marketing: Empresa y comercio. Tesis de master de investigación en economía de la empresa. Universidad de la Rioja.

Morales, O. (2010). Cultura Organizacional y liderazgo en las empresas familiares latinoamericanas. Disponible en: https://www.esan.edu.pe/conexion/actualidad/2011/05/17/cultura-organizacional-y-liderazgo-en-empresas-familiares-latinoamericanas/ Consultado: 28/02/2016.

Quijano G., R. A. Arguelles M., L. A. Sahuí M., J. A. Magaña Medina, D. E. (2015). Experiencia Generacional y Transmisión de Poder Como Elementos de la Cultura Organizacional en Empresas de familia de Campeche, México. Revista Internacional Administración \& Finanzas RIAF, 8 (3), 53-67. Disponible en: file://C:/Users/ USER/Downloads/RIAF-V8N3-2015-4.pdf

Ramírez, V. H., \& Ospina, D. E. (2011). Sucesión en empresas familiares. Análisis desde la teoría de la agencia. Caso: Caldas. Criterio Libre, 9 (15), 193- 212. https://doi.org/10.18041/1900-0642/criteriolibre.2011v9n15.1208

Schubert, T. (2010). Marketing and organisational innovations in entrepreneurial innovation processes and their relation to market structure and firm characteristics. Review of Industrial Organization, 36, 189-212. DOI 10.1007/s11151-010-9243-y

Solarte, C. Solarte, M. Arcos, C. (2017). Ecosistema Emprendedor Colombiano, caso ciudad de Pasto. Pasto, Colombia: Institución Universitaria Centro de Estudios Superiores María Goretti. DOI:10.15658/CESMAG17.01.0807

Terzaghi, F. (2010). Innovación y cultura organizacional. Disponible en: http://fedeterzaghi.blogspot.com/2011/11/ innovacion-y-cultura-organizacional.html Consultado: 1/04/2016.

Utkun, E. y Atilgan, T. (2010). Marketing Innovation in the Apparel Industry: Turkey. Fibres and Textiles. Eastern Europe, 6 (83), 26-31. Disponible en: http://www.fibtex.lodz.pl/pliki/Fibtex_(e093nnrqqtuexu8s).pdf

Vilaseca, J.; Torrent, J.; y Díaz, A. (2002). La Economía del Conocimiento: Paradigma tecnológico y cambio estructural. Un análisis empírico e internacional para la economía española. España: Working paper Series WP02-003. Disponible en: http://www.uoc.edu/in3/dt/20007/index.html Consultado: 11/04/2016.

Yamakawa P. \& Ostos J. (2011). Relación entre innovación organizacional y desempeño organizacional. Revista Universidad \& Empresa (21), 93-115. Disponible en: file://C:/Users/USER/Desktop/1889-6762-1-PB.pdf

Ynzunza Cortés, C. B., \& Izar Landeta, J. M. (2013). Estrategia, Orientación al Mercado y Desempeño Organizacional. Conciencia Tecnológica, 45. Disponible en: https://www.redalyc.org/comocitar.oa?id=94427876002 